La Revue

des Droits

de l'Homme

\section{La Revue des droits de l'homme}

Revue du Centre de recherches et d'études sur les droits fondamentaux

Actualités Droits-Libertés | 2020

\title{
Bilan critique de l'activité des Comités onusiens
}

Situation du système des organes conventionnels des droits de l'Homme, Rapport du Secrétariat général, 2018-2019, A/74/643

\section{Sophie Grosbon}

\section{OpenEdition Journals}

\section{Electronic version}

URL: http://journals.openedition.org/revdh/9309

DOI: $10.4000 /$ revdh.9309

ISSN: 2264-119X

Publisher

Centre de recherches et d'études sur les droits fondamentaux

\section{Electronic reference}

Sophie Grosbon, «Bilan critique de l'activité des Comités onusiens », La Revue des droits de l'homme

[Online], Actualités Droits-Libertés, Online since 10 May 2020, connection on 06 November 2020.

URL : http://journals.openedition.org/revdh/9309 ; DOl : https://doi.org/10.4000/revdh.9309

This text was automatically generated on 6 November 2020 .

Tous droits réservés 


\section{Bilan critique de l'activité des Comités onusiens}

Situation du système des organes conventionnels des droits de l'Homme, Rapport du Secrétariat général, 2018-2019, A/74/643

\section{Sophie Grosbon}

1 En 2014, l'Assemblée générale des Nations Unies a adopté la résolution 68/268 relative $\mathrm{au}$ « renforcement et [à] l'amélioration du fonctionnement de l'ensemble des organes conventionnels des droits de l'Homme ». En vertu de celle-ci, le Secrétaire général de l'ONU doit présenter tous les deux ans un rapport sur le système des organes des traités dans lequel figurent les progrès accomplis en vue d'accroître l'efficacité et l'efficience des travaux de ceux-ci, les difficultés rencontrées ainsi que les nouvelles idées et propositions afférentes.

2 Les organes des traités sont constitués de 10 comités composés d'experts indépendants chargés $\mathrm{du}$ contrôle $\mathrm{du}$ respect des $\mathbf{1 0}$ principaux traités internationaux de droits de l'Homme, chaque organe étant en charge d'une convention particulière.

3 Le document du Secrétariat général « Situation du système des organes conventionnels des droits de l'Homme » (A/74/643) rendu public en janvier dernier constitue donc le $3^{\text {e }}$ rapport d'ensemble exigé par l'Assemblée générale et dresse une forme de bilan d'activité pour la période 2018-2019.

4 Parmi les éléments positifs, on y apprend que, depuis le dernier rapport - datant de fin 2017, le nombre de ratifications des traités, protocoles ou de déclarations d'acceptation des différentes procédures de contrôle a augmenté de 2,7\%. Il est également souligné que les organes ont renforcé les outils permettant de diffuser des connaissances sur les conventions et sur leur mise en œuvre. Ainsi depuis fin 2017, l'équipe de renforcement des capacités a dispensé formations et offert des appuis dans une centaine d'États. Elle a aussi créé un module de formation sur les rapports étatiques à présenter aux organes des traités, accessible en ligne dans les 5 langues officielles de l'ONU et un guide pratique sur le rôle des mécanismes nationaux dans la prévention de la torture. L'index universel des droits de l'Homme, base de données 
des documentations produites par les instances de l'ONU chargées des droits de l'Homme a été mis à jour et amélioré. Une autre base de données visant le suivi au niveau national des recommandations des organes onusiens a été créée. À partir de 2020, les réunions publiques des comités seront diffusées en ligne dans toutes les langues officielles employées par ceux-ci.

5 Parmi les éléments plus négatifs soulevés dans le rapport, deux sujets méritent une attention particulière: le manque de moyen auquel les Comités font face (I) et la question - évoquée dans une moindre mesure - de la désignation des experts indépendants (II).

\section{I/- Le manque cruel de moyens pour accomplir les différentes missions des Comités}

6 Les experts indépendants ne perçoivent pas de salaires pour leurs activités au sein des Comités. L'ONU prend uniquement en charge leurs frais de voyage et leur verse une indemnité journalière pendant leurs réunions et leurs missions. Ils travaillent avec l'appui du Haut-Commissariat aux droits de l'Homme.

7 Difficultés. Le présent rapport insiste sur les difficultés liées au manque de ressources financières, humaines et matérielles auxquelles les Comités ont à faire face dans l'accomplissement de leurs tâches. Il note en particulier que les experts n'ont pas toujours pu utiliser le temps de réunion qui leur était imparti en raison du manque de personnel nécessaire à la préparation de leurs travaux. S'ajoutent à cette difficulté la crise de liquidités que connaît l'ONU et la décision de l'Assemblée Générale prise fin 2017 de réduire de $25 \%$ le budget alloué aux frais de voyage. Ce manque de moyens a des répercussions importantes sur les différentes procédures de contrôle.

8 Arriérés. Certes le retard accumulé dans l'examen des rapports étatiques (procédure de contrôle sur rapport) a été réduit de manière importante (diminution de 20,4\% des arriérés depuis fin 2017). Cependant, avec les capacités actuelles dont disposent les comités, il faudrait plus d'un an pour résorber le stock pendant, et ce, sans compter le fait que 81 \% des États Parties sont en retard dans la remise de leur rapport. Surtout, le nombre de communications individuelles reçues a considérablement augmenté. En 2019, environ 600 communications ont été enregistrées, soit une augmentation de $80 \%$ pour la période 2018-2019. Il faut désormais environ 6 ans aux Comités pour examiner un cas individuel, ce qui a de graves répercussions pour les victimes. En outre, de manière inédite, pendant la période considérée, le Comité pour l'élimination de la discrimination raciale a reçu 3 communications interétatiques, ce qui constitue une charge de travail supplémentaire. Le Sous-comité pour la prévention de la torture dont la principale activité est la visite dans les lieux de privation de liberté n'a pu effectuer que 7 visites en 2019. En 2018-2019, le comité des disparitions forcées a enregistré 345 nouvelles demandes d'interventions d'urgence relatives à la fourniture d'une assistance à la recherche de personnes disparues. Au 31 octobre 2019, il avait clos 65 dossiers (personnes retrouvées vivantes et libérées ou mortes). Son arriéré en augmentation considérable est aujourd'hui de 725 interventions.

9 Ainsi, «les besoins en personnel supplémentaire (...) nécessaires pour appuyer les activités des organes conventionnels nécessitant davantage de main-d'œuvre n'ont pas été pris en compte. Par conséquent, on peut s'attendre à ce que les problèmes de 
capacités persistent, le personnel d'appui actuel étant incapable de produire la documentation destinée à l'examen par les comités dans le temps de réunion approuvé, et à ce que les retards et le temps de réponse augmentent en conséquence » (\$51).

Pistes. Parallèlement à cette insistance sur le manque de moyen, le rapport propose des idées afin de rationaliser et d'harmoniser les différentes procédures. Les comités promeuvent notamment d'ores et déjà une procédure simplifiée de présentation des rapports étatiques, limitée à la synthèse des réponses que les États peuvent fournir à la suite d'une liste de questions sur des points précis que leur adresseraient les organes des traités. Par ailleurs, afin de prendre en compte les besoins des États les plus pauvres dans cette procédure simplifiée, les Comités sont invités à poursuivre le dialogue constructif au niveau régional ou par visioconférence (plutôt que lors de réunions physiques à Genève) et les États à renforcer l'assistance due au titre de la coopération technique. D'une manière générale, l'efficacité pourrait être accrue si les technologies de l'information et de la communication étaient davantage utilisées, notamment en créant une page web interactive commune aux différents comités pour accéder facilement aux documentations et éviter les doublons.

11 D'autres difficultés sont pointées du doigt dans le rapport, notamment - avec euphémisme parfois - celles relatives à la composition des Comités.

\section{II/- Une composition perfectible des Comités}

Représentativité. Alors que les conventions et la résolution 68/268 insistent sur l'exigence de représentativité des membres des Comités dont la composition doit tenir compte de l'équilibre entre les sexes, d'une répartition géographique équitable, d'une représentation des différentes civilisations et systèmes juridiques et de la participation d'experts handicapés, le rapport relève le manque de parité des comités puisque pour un certain nombre d'entre eux seuls « deux membres ne sont pas du même sexe que les autres » (\$ 59). Le rapport aurait pu souligner plus nettement la très faible proportion de femmes parmi les experts indépendants (à l'exception du Comité pour l'élimination de la discrimination faite aux femmes qui ne comporte qu'un homme et du Comité pour l'élimination de la discrimination raciale qui présente une représentation paritaire). Il aurait également pu examiner plus précisément les autres éléments de représentativité.

Légitimité. Mais surtout le rapport insiste sur la procédure de sélection des experts et sur la nécessité de garantir le plus haut niveau de compétence, la grande moralité et l'indépendance des membres désignés. Pour cela, les États sont invités, avant de proposer des candidats, à mettre en place au niveau national des concours ou d'autres procédures indépendantes permettant de vérifier les antécédents et le mérite de chacun. De telles pratiques restent à l'heure actuelle exceptionnelles. Ainsi, souligne le rapport, "la pratique des États qui présentent des listes où le nombre de candidats correspond exactement au nombre de postes à pourvoir n'encourage pas des élections véritablement pluralistes » (\$59) (c'est le moins que l'on puisse dire...) et « devrait être fortement découragée afin d'accroitre la probabilité que les candidats soient élus au mérite » (\$71).

En vertu de la résolution 68/268, l'efficacité des mesures prises depuis 2014 en vue du renforcement du système des organes des traités sera examinée dans sa totalité en cette année 2020. Il s'agira alors de garantir la viabilité du système onusien de 
protection des droits de l'Homme et de prendre les mesures supplémentaires nécessaires en vue de son amélioration.

Assemblée générale des Nations Unies, Situation du système des organes conventionnels des droits de l'homme, Rapport du Secrétaire général, A/74/643, 23 janvier 2020.

Les Lettres « Actualités Droits-Libertés » (ADL) du CREDOF (pour s'y abonner) sont accessibles sur le site de la Revue des Droits de l'Homme (RevDH) - Contact

\section{ABSTRACTS}

Le rapport "Situation du système des organes conventionnels des droits de l'Homme » décrit pour la période 2018-2019 les progrès accomplis en vue d'accroître l'efficacité des travaux des comités onusiens et les difficultés rencontrées, relatives notamment au manque de moyens.

\section{AUTHOR}

\section{SOPHIE GROSBON}

Maitresse de Conférences en Droit privé à l'Université Paris Nanterre 